\title{
Advances in Personalized Therapeutic Approaches in Myelodysplastic Syndromes
}

Presented by Rafael Bejar, MD, PhD

\begin{abstract}
Often unrecognized and underdiagnosed, myelodysplastic syndromes (MDS) are a rare group of cancers in which the bone marrow fails to produce sufficient healthy blood cells. Although patients with lower-risk MDS can live for $>5$ years, those with high-risk disease that evolves into acute myeloid leukemia is associated with significantly lower overall survival. At the NCCN 2019 Annual Congress: Hematologic Malignancies, Dr. Rafael Bejar summarized current standard treatment options for patients with MDS and discussed the importance of genetic testing to identify mutations that may impact treatment. Finally, Dr. Bejar described emerging personalized treatment strategies for the management of this disease. J Natl Compr Canc Netw 2019;17(11.5):1444-1447 doi: 10.6004/jnccn.2019.5032
\end{abstract}

\section{Optimizing Therapy for Individual Patients} Myelodysplastic syndromes (MDS) are a group of bone marrow failure disorders characterized by ineffective hematopoiesis. For many patients with lower-risk MDS, life expectancy can exceed 5 years. However, for those with higher-risk MDS that evolves into acute myeloid leukemia (AML), overall survival (OS) is significantly reduced. At the NCCN 2019 Annual Congress: Hematologic Malignancies, Rafael Bejar, MD, PhD, Associate Professor, UC San Diego Moores Cancer Center, discussed current standard-of-care treatment in the NCCN Guidelines for MDS, which is split into 2 separate algorithms addressing lower-risk and higher-risk MDS. He also described emerging personalized treatment strategies for the management of MDS, including the increased role of genetic testing to identify high-impact mutations.

As Dr. Bejar explained, researchers have identified dozens of different somatic genetic mutations that can lead to MDS. Most patients will have $>1$ mutation and a unique pattern of mutation, as genetic profiles in MDS demonstrate a great deal of heterogeneity. "No two patients with MDS are truly alike," said Dr. Bejar. "Unfortunately, somatic mutation is not a static process but evolves over time. Additional mutations will accumulate, often leading to a more profound phenotype of worsening cytopenias, and approximately $30 \%$ of patients will eventually experience progression to secondary AML."

\section{Personalized Risk Assessment}

Using a case study involving a 65-year-old healthy man who presented with fatigue and shortness of breath, Dr. Bejar illustrated how genetic testing is used to generate a personalized risk assessment that will inform treatment. After a bone marrow biopsy revealed a hypercellular bone marrow with clear dysplasia, $1 \%$ blasts in the bone marrow aspirate, dysplasia in all 3 cell types, and a karyotype with isolated del(5q) —elements that are characteristic of MDS - Dr. Bejar used the Revised International Prognostic Scoring System (IPSS-R) to assess the patient's risk. ${ }^{1}$ According to the IPSS-R, the patient was in the low-risk group. Patients with an IPSS-R score $\leq 3.5$ are classified as having lower-risk disease, whereas those with a score $>3.5$ are considered to have higher-risk disease (Figure 1).

"The different groups in the IPSS-R stratify quite nicely, with big differences in OS between patients in the highest-risk group and those in the lowest-risk group," said Dr. Bejar, who noted that median OS in the low-risk group is approximately 5 years. "Accordingly, the NCCN Guidelines for MDS are divided into 2 almost completely separate algorithms, with one treatment approach for lowerrisk patients and another for those with higher-risk MDS."

As Dr. Bejar explained, for patients with isolated $\operatorname{del}(5 q)$ and lower-risk MDS, the treatment of choice is lenalidomide (Figure 2). Patients in this population frequently respond to treatment (overall response rate, $50 \%-80 \%$ ), and responses are typically deep and durable. On average, patients experience responses lasting $>1.5$ years, with many responses exceeding $>2$ years.

For patients without del(5q) deletion, however, alternative options, such as erythropoiesis-stimulating agents (ESAs), are usually considered first. According to Dr. Bejar, patients with very low hemoglobin levels and who are heavily transfusion-dependent are unlikely to respond to ESAs. For those with endogenous erythropoietin levels $<200 \mathrm{U} / \mathrm{L}$ and who are mildly transfusion-dependent, an 

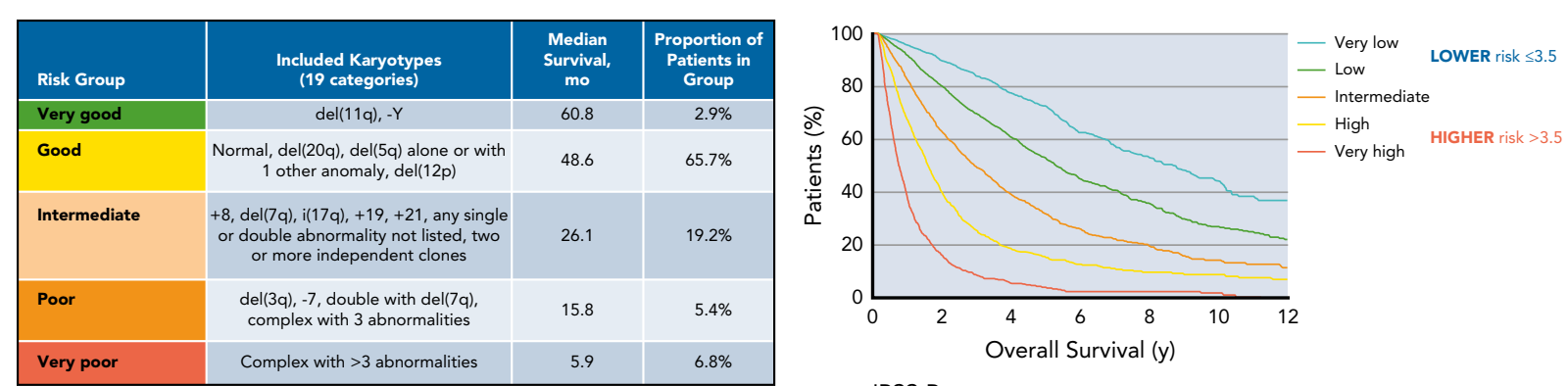

IPSS-R score:

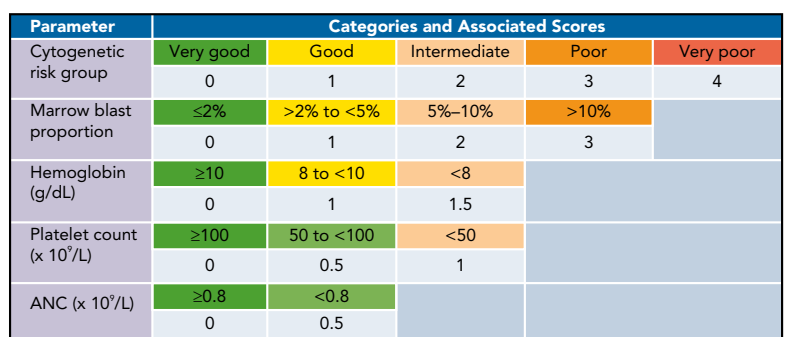

- 1 point for del(5q)

- 0 points for blasts

- 1 point for low hemoglobin

- 0 points for platelet count and ANC

Total $=2$ points $\rightarrow$ LOW risk group

\begin{tabular}{|l|l|c|c|c|}
\hline Risk Group & Points & Patients & $\begin{array}{c}\text { Median Survival, } \\
\mathbf{y}\end{array}$ & $\begin{array}{c}\text { Time Until 25\% of } \\
\text { Patients Develop } \\
\text { AML, } \mathbf{y}\end{array}$ \\
\hline Very low & $\leq 1.5$ & $19 \%$ & 8.8 & Not reached \\
\hline Low & $>1.5-3$ & $38 \%$ & 5.3 & 10.8 \\
\hline Intermediate & $>3-4.5$ & $20 \%$ & 3.0 & 3.2 \\
\hline High & $>4.5-6$ & $13 \%$ & 1.6 & 1.4 \\
\hline Very high & $>6$ & $10 \%$ & 0.8 & 0.7 \\
\hline
\end{tabular}

Figure 1. Revised IPSS-R.

Abbreviations: ANC, absolute neutrophil count; AML, acute myeloid leukemia; IPSS-R, Revised International Prognostic Scoring System.

Adapted from Schanz J, Tüchler H, Solé F, et al. New comprehensive cytogenetic scoring system for primary myelodysplastic syndromes (MDS) and oligoblastic acute myeloid leukemia after MDS derived from an international database merge. J Clin Oncol 2012;30:820-829; and Greenberg PL, Tuechler H, Schanz, J, et al. Revised International Prognostic Scoring System for myelodysplastic syndromes. Blood 2012;120:2454-2465.

ESA will likely increase hemoglobin levels to the point of transfusion independence.

A new approach targeting transforming growth factor (TGF)- $\beta$ signaling is on the horizon. A phase II study of luspatercept, a novel fusion protein that blocks TGF- $\beta$ superfamily inhibitors of erythropoiesis, showed a significant reduction in transfusion requirements among patients with anemia due to lower-risk MDS with ring sideroblasts. ${ }^{2}$ Furthermore, time-to-response was fairly rapid and the response was sustained for the 24-week analysis period. Although not yet FDA-approved, luspatercept could be approved in 2020, said Dr. Bejar.

\section{High-Impact Genetic Mutations}

Although the IPSS-R is the gold standard for defining clinical risk in patients with MDS, it does consider somatic mutations, said Dr. Bejar, who noted that certain mutations can portend a worse prognosis. An analysis of $>400$ patients stratified by IPSS-R risk group showed that such adverse mutations were present in approximately $20 \%$ of patients with very-low-risk MDS (Figure 3 ) ${ }^{3}$ These patients had a median OS that was nearly one-third shorter than that of low-risk patients without these mutations. For patients with low and intermediate IPSS-R risk, median OS was approximately $50 \%$ shorter compared with those without mutations. A separate analysis of nearly 2,000 patients with MDS sequenced at centers around the world noted that patients with a greater number of somatic mutations had inferior OS. ${ }^{4}$ "I'm not advocating that we necessarily count mutations to determine a patient's prognosis, given that the presence of certain mutations is worse than others," said Dr. Bejar. "Some mutations may simply be neutral with regard to prognosis."

This collaborative analysis with the International Working Group for Prognosis in MDS found that $42 \%$ of patients with MDS have a mutation that is associated with worse prognosis than predicted by the IPSS-R. ${ }^{4}$ These adverse, somatically mutated genes included $A S X L 1, R U N X 1, E Z H 2, C B L$, and NRAS, among others. "It seems like we're underestimating risk in a substantial number of patients," Dr. Bejar observed. "If a patient is on the intermediate-risk border with one of these adverse-risk mutations, I would argue that their clinical behavior is going to be similar to that of traditional, higher-risk individuals." 


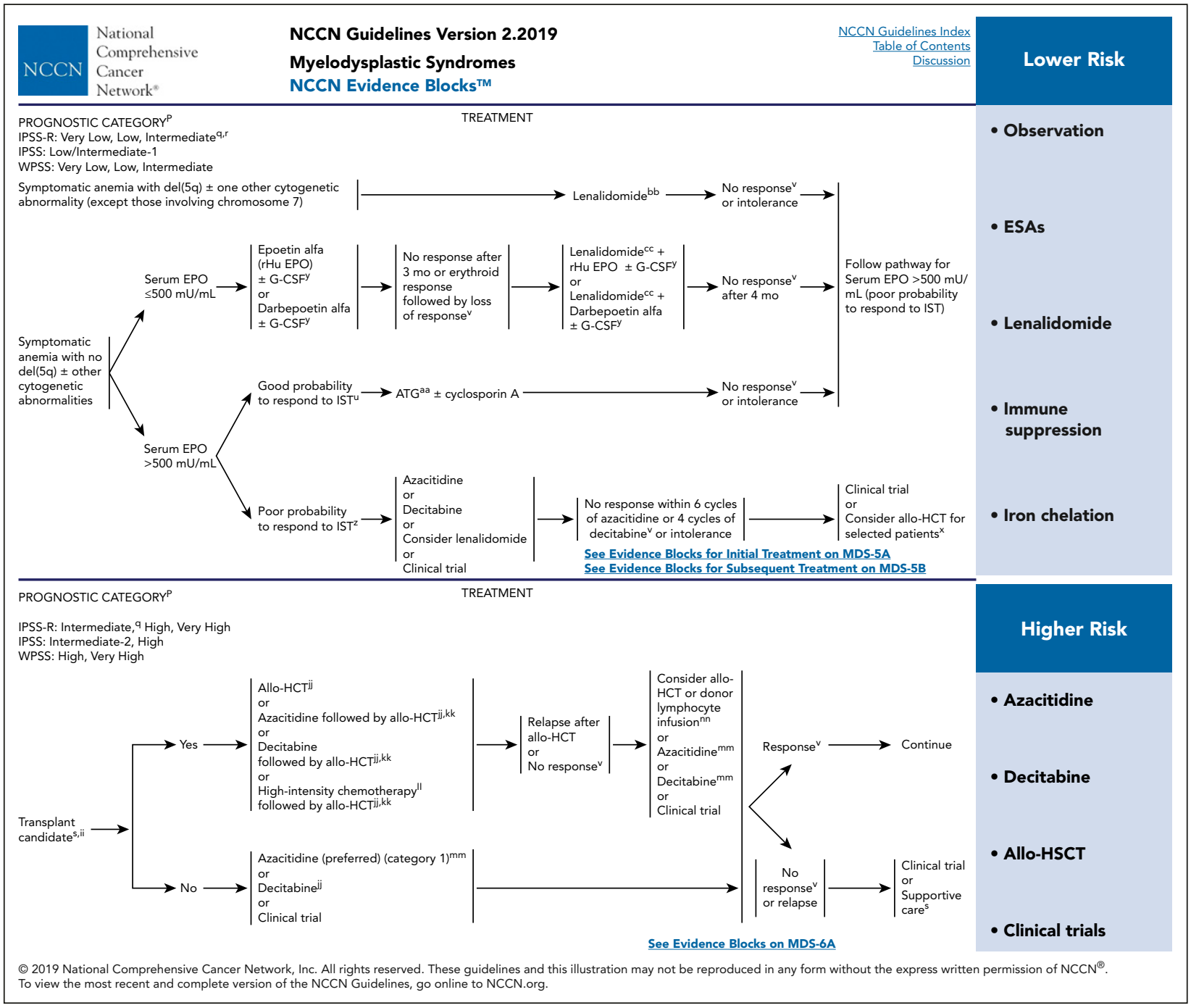

Figure 2. Treatment for myelodysplastic syndromes is risk-stratified in the NCCN Guidelines for Myelodysplastic Syndromes. Version 2.209. To view the most recent version, visit NCCN.org.

Abbreviations: allo-HSCT, allogeneic hematopoietic stem cell transplantation; ESAs, erythropoiesis-stimulating agents.

\section{TP53 Mutations}

One genetic lesion that has recently come under greater scrutiny is mutation in TP53, a common resistance marker for patients whose disease progresses on lenalidomide. As Dr. Bejar discussed, data suggest that mutations of TP53 are particularly adverse in almost all settings, including patients who are undergoing transplantation. In a study of $>$ 1,500 patients, TP53 mutations were shown to be associated with approximately $50 \%$ shorter long-term survival when compared with those without the mutation. ${ }^{5}$ Additionally, even younger patients with this mutation had notably poor outcomes.

"At our center, we don't perform traditional transplants for patients with TP53 mutations anymore," said Dr. Bejar. "We try to perform a transplant that includes something else that we think could increase the chances of long-term survival." One potentially promising option for patients with TP53-mutated disease is intensive therapy with decitabine. Welch et $\mathrm{al}^{6}$ identified a veryhigh-response rate for patients who received high-dose decitabine and had TP53 mutations in the setting of MDS or AML. "Strikingly, by the fourth cycle of treatment, investigators actually observed cytoreduction of the TP53-mutant clone," said Dr. Bejar. "This suggests that if the transplant is timed appropriately after treatment, patients might have less residual disease and may have a longer time to relapse, allowing their graft to take hold. This approach is now being tested in clinical studies."

Another option for patients who are unable to undergo a traditional transplant is the experimental drug APR-246, which is believed to stabilize mutant forms 


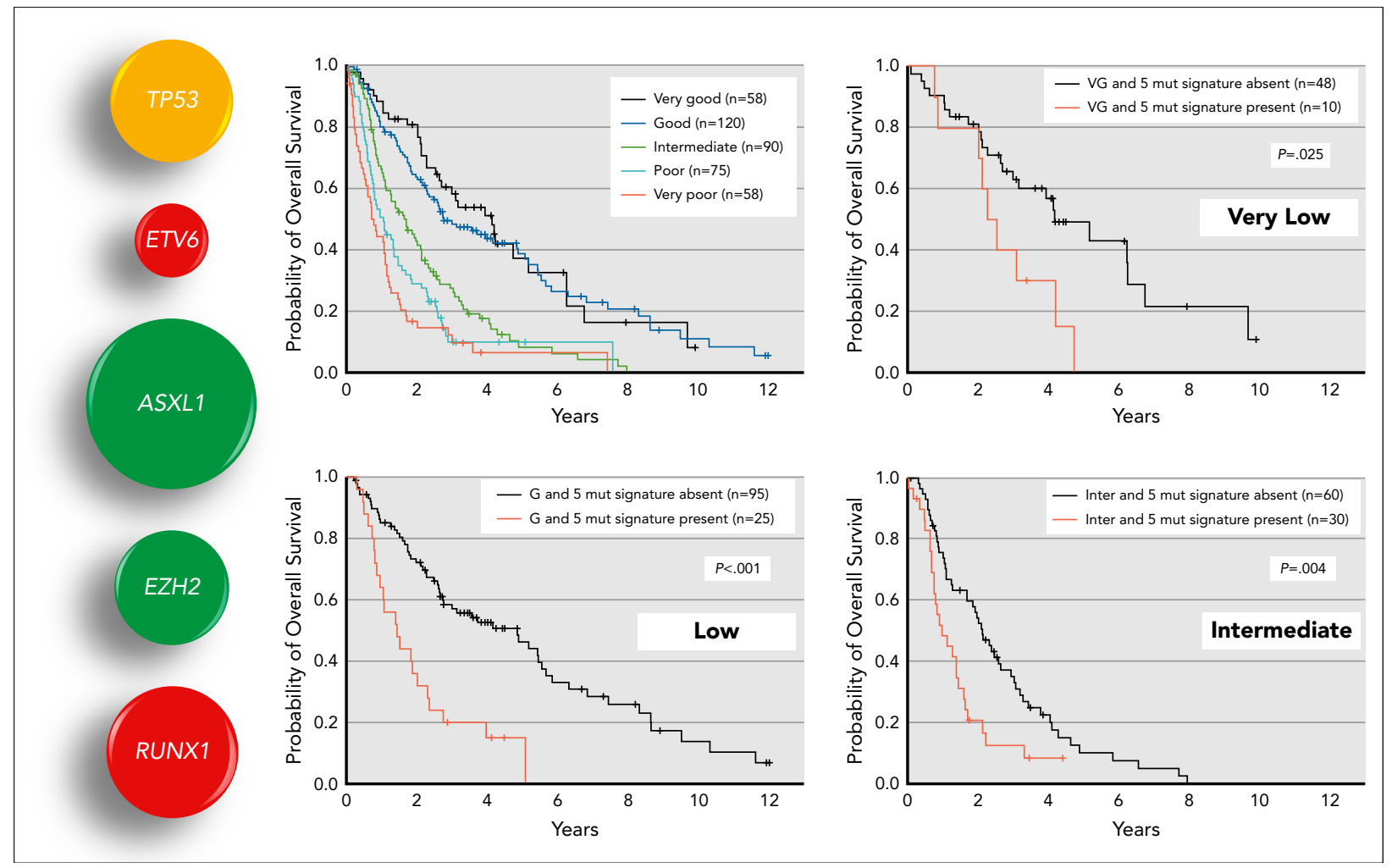

Figure 3. Impact of mutations by IPSS-R group.

Abbreviations: G, good; Inter, intermediate; mut, mutation; VG, very good.

Adapted from Bejar R, Steensma DP. Recent developments in myelodysplastic syndromes. Blood 2014;124:2793-2803.

of TP53, thereby allowing the mutated gene to carry out its normal endogenous function. Data from phase I and II studies have demonstrated both a high rate of response and durable responses through the follow-up period. ${ }^{7}$ Nevertheless, said Dr. Bejar, APR-246 will probably not keep patients in long-term remission on its own, but will be combined with other agents in future studies.

Lastly, Dr. Bejar discussed the possibility of a personalized approach involving use of immunotherapeutic agents in MDS. Studies at MD Anderson Cancer Center are currently combining PD-1 and PD-L1 inhibitors with hypomethylating agents. At UC San Diego, studies are currently evaluating a neoantigen-targeted approach using patients' own $\mathrm{T}$ cells selected for their ability to recognize tumor-associated mutated peptides. "Our hope is that these approaches could produce longlasting immunologic responses in these patients," said Dr. Bejar.

Disclosures: Dr. Bejar has disclosed that he is a scientific advisor for Celgene, NeoGenomics, AbbVie, Astex, and Daiichi-Sankyo; has received grant/research support from Takeda and Celgene; and is on the advisory board for Celgene/Acceleron

Correspondence: Rafael Bejar, MD, PhD, UC San Diego Moores Cancer Center, 3855 Health Sciences Drive, MC 0820, La Jolla, CA 92093. Email: rabejar@ucsd.edu

\section{References}

1. Schanz J, Tüchler H, Solé F, et al. New comprehensive cytogenetic scoring system for primary myelodysplastic syndromes (MDS) and oligoblastic acute myeloid leukemia after MDS derived from an international database merge. J Clin Oncol 2012;30:820-829.

2. Platzbecker U, Germing U, Götze KS, et al. Luspatercept for the treatment of anaemia in patients with lower-risk myelodysplastic syndromes (PACEMDS): a multicentre, open-label phase 2 dose-finding study with long-term extension study. Lancet Oncol 2017;18:1338-1347.

3. Bejar R, Steensma DP. Recent developments in myelodysplastic syndromes. Blood 2014;124:2793-2803.

4. Bejar R, Papaemmanuil E, Haferlach T, et al. Somatic mutations in MDS patients are associated with clinical features and predict prognosis

independent of the IPSS-R: analysis of combined datasets from the International Working Group for Prognosis in MDS-Molecular Committee [abstract]. Blood 2015;126:Abstract 637.

5. Lindsley RC, Saber W, Mar BG, et al. Prognostic mutations in myelodysplastic syndrome after stem-cell transplantation. N Engl J Med 2017;376: 536-547.

6. Welch JS, Petti AA, Miller CA, et al. TP53 and decitabine in acute myeloid leukemia and myelodysplastic syndromes. N Engl J Med 2016;375: 2023-2036.

7. Bykov VJ, Zhang Q, Zhang M, et al. Targeting of mutant p53 and the cellular redox balance by APR-246 as a strategy for efficient cancer therapy. Front Oncol 2016;6:21. 